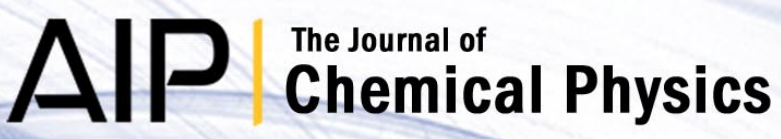

Capturing static and dynamic correlations by a combination of projected Hartree-Fock and density functional theories

Alejandro J. Garza, Carlos A. Jiménez-Hoyos, and Gustavo E. Scuseria

Citation: J. Chem. Phys. 138, 134102 (2013); doi: 10.1063/1.4796545

View online: http://dx.doi.org/10.1063/1.4796545

View Table of Contents: http://jcp.aip.org/resource/1/JCPSA6/v138/i13

Published by the American Institute of Physics.

\section{Additional information on J. Chem. Phys.}

Journal Homepage: http://jcp.aip.org/

Journal Information: http://jcp.aip.org/about/about_the_journal

Top downloads: http://jcp.aip.org/features/most_downloaded

Information for Authors: http://jcp.aip.org/authors

\section{ADVERTISEMENT}

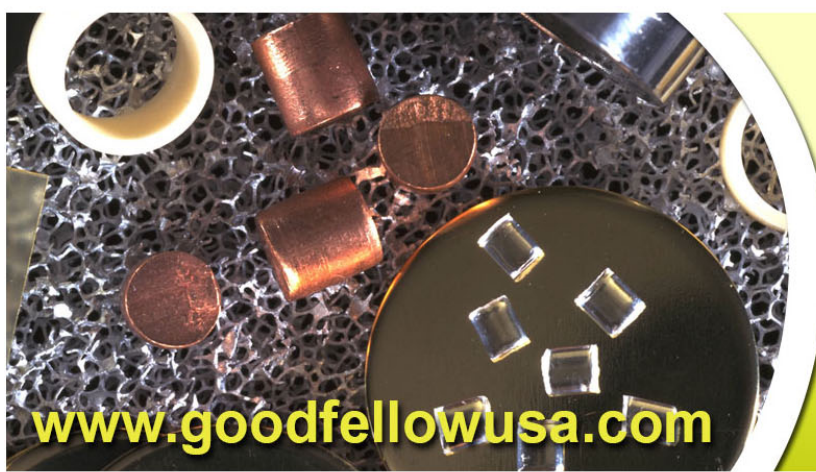




\title{
Capturing static and dynamic correlations by a combination of projected Hartree-Fock and density functional theories
}

\author{
Alejandro J. Garza, ${ }^{1}$ Carlos A. Jiménez-Hoyos, ${ }^{1}$ and Gustavo E. Scuseria ${ }^{2}$ \\ ${ }^{1}$ Department of Chemistry, Rice University, Houston, Texas 77251-1892, USA \\ ${ }^{2}$ Department of Chemistry and Department of Physics and Astronomy, Rice University, Houston, \\ Texas 77251-1892, USA
}

(Received 29 January 2013; accepted 8 March 2013; published online 1 April 2013)

\begin{abstract}
This paper explores the possibility of combining projected Hartree-Fock and density functional theories for treating static and dynamic correlations in molecular systems with mean-field computational cost. The combination of spin-projected unrestricted Hartree-Fock (SUHF) with the TPSS correlation functional (SUHF+TPSS) yields excellent results for non-metallic molecular dissociations and singlet-triplet splittings. However, SUHF+TPSS fails to provide the qualitatively correct dissociation curve for the notoriously difficult case of the chromium dimer. By tuning the TPSS correlation parameters and adding complex conjugation symmetry breaking and restoration to SUHF, the right curve shape for $\mathrm{Cr}_{2}$ can be obtained; unfortunately, such a combination is found to lead to overcorrelation in the general case. (C) 2013 American Institute of Physics. [http://dx.doi.org/10.1063/1.4796545]
\end{abstract}

\section{INTRODUCTION}

To date, no method is known to have the ability to simultaneously describe weak (or dynamic) and strong (or static) correlations at low computational cost. However, an efficient projected Hartree-Fock (PHF) methodology with mean-field computational cost has recently been proposed and implemented by our research group. ${ }^{1}$ The most attractive feature of spin-PHF is that it is capable of dealing with strong correlations arising from spin fluctuations while retaining the low computational scaling of the regular Hartree-Fock method. ${ }^{2}$ On the other hand, density functional theory (DFT) has a well established reputation because of its ability to account for some static and weak correlations via exchange and correlation functionals, respectively, ${ }^{3}$ all within mean-field computational cost too. It then seems quite logical to attempt to combine PHF and DFT correlation functionals in a scheme taking advantage of the particular abilities of each method. Assuming that PHF accounts for all static correlations, one could, for example, compute the PHF wavefunction and then use the resulting density for calculating the residual dynamical correlations from DFT.

The idea of adding DFT correlation functionals to methods that can capture static correlation has been explored in the past. ${ }^{4-11}$ Blends of DFT with complete active space $^{12-14}$ (CAS), configuration interaction ${ }^{15}(\mathrm{CI})$, multiconfiguration self-consistent field ${ }^{16}$ (MCSCF), and constrainedpairing mean-field theory ${ }^{17,18}$ (CPMFT) have emerged in the literature during the last decade. Compared to these methods, PHF has the advantage of being a "black box" tool in the sense that it does not require the specification of the number of active orbitals or electrons from the user. Furthermore, PHF has a much lower computational scaling than CI, MCSCF, or CAS, and as shown below, PHF+DFT does not seem to require the inclusion of DFT exchange in molecules near equilibrium, as occurs with CPMFT. ${ }^{18}$
There are, however, two central issues that one must consider before using such a PHF+DFT scheme: symmetry inconsistencies and double counting of correlations. The first one refers to the fact that PHF yields a wavefunction that respects the symmetries of the molecular Hamiltonian, while modern DFT functionals are designed to work with broken symmetry densities. ${ }^{19}$ The second problem is basically the possibility of overcorrelation; the PHF wavefunction may already contain dynamic correlations which would then lead to double counting when adding residual correlations from DFT correlation functionals. These two problems are not unique to PHF and are also encountered in other multireference (MR) DFT procedures. ${ }^{12,15,16}$

In this paper, we address the above issues and present the results of our implementation for a simple PHF+DFT model. Our aim is to demonstrate that PHF+DFT is a promising, computationally economic tool for handling molecular systems in which both dynamic and static correlations are important. We begin by explaining in detail the problem of symmetry discrepancies between PHF and DFT, and present an approach to solve it by means of alternative densities. These alternative densities are then used in a single-shot postPHF calculation for adding residual correlations via DFT. The rest of the paper is dedicated to describe the details of our implementation and the results of benchmark calculations.

\section{THEORY AND METHODS}

\section{A. Alternative densities}

The symmetry dilemma of DFT and the rationale behind the need of using alternative densities in PHF+DFT can be summarized as follows: the most successful DFT approach to date has been Kohn-Sham (KS) DFT. ${ }^{20}$ All KS-DFT functionals are approximations to a formally exact functional which depend only on the total electronic density $\gamma(r)=\gamma_{\alpha}(r)$ $+\gamma_{\beta}(r) .{ }^{21}$ However, methods that depend only on $\gamma(r)$ yield 
poor quality results if strong correlations are present. This problem is alleviated by using functionals which depend on both $\gamma_{\alpha}(r)$ and $\gamma_{\beta}(r)$ (or, equivalently, $\gamma(r)$ and the spin density $\gamma_{z}(r)$ ) and breaking spin symmetry. In consequence, modern functionals have been designed to work with brokensymmetry densities ${ }^{19,22}$ (this technique is sometimes referred to as unrestricted or spin DFT). Since PHF yields symmetryadapted densities, we must define and use alternative densities if we are to take advantage of the vast resources currently available from KS-DFT methods.

Perhaps the most rigorous way around this symmetry dilemma is the one provided by Perdew et al. ${ }^{19}$ According to this interpretation, rather than yielding physically accurate values for $\gamma_{\alpha}(r)$ and $\gamma_{\beta}(r)$, DFT yields correct values for $\gamma(r)$ and the on-top pair density $P_{2}(r)$, the probability of finding two electrons at the same point in space. This view is perfectly valid as the Hohenberg-Kohn theorem ${ }^{21}$ makes no reference to the individual densities $\gamma_{\alpha}(r)$ and $\gamma_{\beta}(r)$, but only to $\gamma(r)$ and the energy, which is also related to $P_{2}(r)$ because of contributions from interelectronic repulsions.

Following this philosophy, we can create alternative densities defined as $\gamma_{ \pm}(r)=\left(\gamma(r) \pm \gamma_{z}(r)\right) / 2$ by substituting $\gamma_{\alpha}(r)$ and $\gamma_{\beta}(r)$ by $\gamma_{+}(r)$ and $\gamma_{-}(r)$ in the expression of $P_{2}(r)$ for a single-determinant state, $P_{2}(r)=\gamma_{\alpha}(r) \gamma_{\beta}(r) .{ }^{23,24}$ This procedure yields the densities used by Moscardó and SanFabián ${ }^{8}$

$$
\gamma_{ \pm}(r)=\frac{1}{2} \gamma(r) \pm \frac{1}{2} \sqrt{\gamma(r)^{2}-4 P_{2}(r)},
$$

where the term $\gamma_{z}(r)=\sqrt{\gamma(r)^{2}-4 P_{2}(r)}$ is basically an alternative spin density. Unfortunately, this approach has two pressing drawbacks in practical applications. The first one is related to the cost of computing $P_{2}(r)$; in general, it is obtained from the pair density $P_{2}\left(r, r^{\prime}=r\right)=P_{2}(r)$,

$$
P_{2}\left(r, r^{\prime}\right)=\sum_{i j k l} \Gamma_{i j}^{k l} \varphi_{i}^{*}(r) \varphi_{j}^{*}\left(r^{\prime}\right) \varphi_{k}(r) \varphi_{l}\left(r^{\prime}\right),
$$

where $\varphi_{i}$ represents a spin orbital and $\Gamma$ is the reduced twoparticle density matrix. Knowledge of $P_{2}(r)$ requires then the evaluation and storage of $\Gamma$, a computationally demanding procedure. The second drawback is that, for densities formed from wavefunctions of multireference character, such as PHF wavefunctions, it is possible that $4 P_{2}(r)>\gamma(r)^{2}$ leading to unphysical complex densities in Eq. (1) and a formally imaginary spin density. ${ }^{25}$ Because of these complications, we have avoided in this work the use of the alternative densities defined by Eq. (1).

The simplest and most economic approach to obtain usable alternative densities in PHF+DFT would be to form densities from an unprojected state that breaks spin symmetry. In the spin-projected PHF method (SUHF), we have a variationally optimized Slater determinant $|\Phi\rangle$ which breaks spin symmetry, and a projected state $|\Psi\rangle=\mathcal{N} \hat{P}|\Phi\rangle$, where $\hat{P}$ is the spin-projection operator recovering the components of $|\Phi\rangle$ having a specific eigenvalue of $\hat{S}^{2}$ and $\mathcal{N}$ is a normalization factor. As usual, we can use the one-particle reduced density matrix $\gamma$

$$
\gamma=\left(\begin{array}{cc}
\gamma_{\alpha} & 0 \\
0 & \gamma_{\beta}
\end{array}\right)
$$

where $\gamma_{i j}=\left\langle\Psi\left|\hat{a}_{j}^{\dagger} \hat{a}_{i}\right| \Psi\right\rangle$, to get $\gamma_{\alpha}(r)$ and $\gamma_{\beta}(r)$ from

$$
\gamma_{\sigma}\left(r, r^{\prime}\right)=\sum_{i j}\left(\gamma_{\sigma}\right)_{i j} \varphi_{\sigma i}^{*}(r) \varphi_{\sigma j}\left(r^{\prime}\right)
$$

by setting $r^{\prime}=r$ (here, $\varphi_{\sigma i}^{*}(r)$ is a spatial orbital of $\sigma$ spin). Alternative densities can be formed if, rather than forming $\gamma_{\sigma}(r)$ using the elements $\gamma_{i j}$, we form instead a deformed density $\varrho_{\sigma}(r)$ from the matrix $\varrho_{i j}=\left\langle\Phi\left|\hat{a}_{j}^{\dagger} \hat{a}_{i}\right| \Phi\right\rangle$. The resulting $\varrho_{\alpha}(r)$ and $\varrho_{\beta}(r)$ components break spin symmetry and are compatible with DFT correlation functionals. For details regarding the projection operators used here and how to build the PHF density matrices, see Refs. 1 and 26.

A second viable possibility is to define alternative densities based only on the spinless (or charge) first-order density matrix $\gamma\left(r, r^{\prime}\right)=\gamma_{\alpha}\left(r, r^{\prime}\right)+\gamma_{\beta}\left(r, r^{\prime}\right)$. Takeda et al. ${ }^{13}$ as well as Pérez-Jiménez and Pérez-Jordá ${ }^{16}$ have favored this approach in their implementations of MR+DFT methods. Pérez-Jiménez and Pérez-Jordá define the densities $\rho_{>}(r)$ and $\rho_{<}(r)$ in terms of the natural orbitals $\psi_{i}(r)$ and occupation numbers of $\gamma\left(r, r^{\prime}\right), n_{i}$ (i.e., eigenvalues of $\left.\gamma_{c}=\gamma_{\alpha}+\gamma_{\beta}\right)^{27}$

$$
\begin{gathered}
\rho_{>}(r)=\sum_{n_{i} \geq 1}\left(n_{i}-1\right)\left|\psi_{i}(r)\right|^{2}, \\
\rho_{<}(r)=\sum_{n_{i} \geq 1}\left|\psi_{i}(r)\right|^{2}+\sum_{n_{i}<1} n_{i}\left|\psi_{i}(r)\right|^{2} .
\end{gathered}
$$

Similar densities have also been derived using the density of unpaired electrons $D\left(r, r^{\prime}\right)$ of Takatsuka et al. ${ }^{28}$

$$
D\left(r, r^{\prime}\right)=\sum_{i} n_{i}\left(2-n_{i}\right) \psi_{i}^{*}\left(r^{\prime}\right) \psi_{i}(r) .
$$

Such is the case of the $u_{A}(r)$ and $u_{B}(r)$ densities defined by Staroverov and Davidson ${ }^{29}$

$$
\begin{aligned}
& u_{A}(r)=\frac{1}{2} \sum_{i} n_{i}\left(1+n_{i}\left(2-n_{i}\right)\right)\left|\psi_{i}(r)\right|^{2}, \\
& u_{B}(r)=\frac{1}{2} \sum_{i} n_{i}\left(1-n_{i}\left(2-n_{i}\right)\right)\left|\psi_{i}(r)\right|^{2},
\end{aligned}
$$

which can be written in their matrix representation as $u_{A}=\gamma_{c}(1+D) / 2$ and $u_{B}=\gamma_{c}(1-D) / 2$, where $\gamma_{c}=\gamma_{\alpha}$ $+\gamma_{\beta}$. Although a more natural definition for alternative densities based on $D\left(r, r^{\prime}\right)$ would have been $u_{A}^{\prime}=\left(\gamma_{c}+D\right) / 2$ and $u_{B}^{\prime}=\left(\gamma_{c}-D\right) / 2$, this prescription allows the appearance of spurious negative densities. This problem arises from the definition of $D\left(r, r^{\prime}\right)$ which does not enforce $\operatorname{Tr}(D) \leq \operatorname{Tr}\left(\gamma_{c}\right)$, i.e., it allows the number of effectively unpaired electrons to be larger than the actual number of electrons. An alternative definition for the density of unpaired electrons $U\left(r, r^{\prime}\right)$ avoids this problem: ${ }^{30}$

$$
U\left(r, r^{\prime}\right)=\sum_{i} \min \left\{n_{i}, 2-n_{i}\right\} \psi_{i}^{*}\left(r^{\prime}\right) \psi_{i}(r) .
$$

We note here that the densities defined by Eqs. (5) and (6) can also be defined in terms of the alternative $U\left(r, r^{\prime}\right)$ above as $\rho_{<}=\left(\gamma_{c}+U\right) / 2$ and $\rho_{>}=\left(\gamma_{c}-U\right) / 2$.

Figure 1 maps the occupation numbers of $\gamma\left(r, r^{\prime}\right), n_{i}$, onto those of the alternative densities $u_{A}, u_{B}, \rho_{>}$and $\rho_{<}$. 


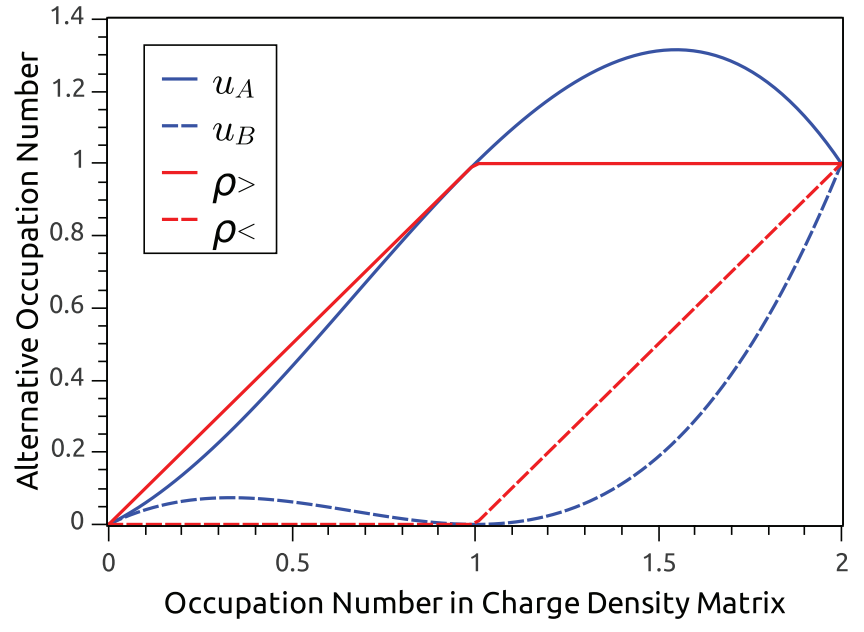

FIG. 1. Occupation numbers of alternative densities in terms of the eigenvalues of the charge density matrix.

Although these two sets of alternative densities are quite different from each other, both satisfy the physical constraints of non-negativity and adding to the total density. In this work, we have used these densities as well as the deformed densities $\varrho_{\alpha}$ and $\varrho_{\beta}$ for our PHF+DFT methodology, which we now proceed to describe.

\section{B. Description of the PHF+DFT method}

In our PHF+DFT scheme, the exact energy $E$ is approximated as

$$
E \approx E^{\mathrm{PHF}}+E_{c}^{\mathrm{DFT}},
$$

where $E^{\mathrm{PHF}}$ is the PHF energy obtained via a self-consistent procedure, and $E_{c}^{\mathrm{DFT}}$ is the correlation energy from a singlepoint calculation using a DFT functional that takes as arguments the alternative densities described in Sec. II A. To avoid the introduction of self-interaction error ${ }^{31-34}$ when calculating residual correlations with DFT, we have chosen the TPSS functional ${ }^{37}$ to compute $E_{c}^{\mathrm{DFT}}$. Additionally, the TPSS correlation functional has two parameters set as $C(0,0)=0.53$ and $d=2.8$ hartree $^{-1}$ that can be reoptimized for our purposes without affecting the self-interaction correction. The original values of $C(0,0)$ and $d$ were chosen so that the TPSS energy of jellium would remain unchanged with respect to its PBE $\mathrm{GGA}^{35,36}$ values. However, it is reasonable to modify $C(0,0)$ and $d$ for PHF+DFT because here we are interested only in residual correlations via alternative densities. Additional details about TPSS and these parameters can be consulted in the original paper. ${ }^{37}$

In PHF methods, the larger the number of symmetries broken and restored, the more correlations are captured. By using SUHF (which restores $\hat{S}^{2}$ symmetry only) to obtain $E^{\mathrm{PHF}}$, and optimizing the parameters $C(0,0)$ and $d$ for performance in SUHF+TPSS, we expect to reduce the possibility of overcorrelation. Specifically, $C(0,0)$ and $d$ were chosen so that SUHF+TPSS would fit the exact dissociation curve for the hydrogen molecule using the cc-pVTZ basis. These values are then used in all other benchmarks, except when explicitly mentioned. Although we here focus on SUHF+TPSS, we also discuss a few results with KSUHF+TPSS (that breaks and restores collinear spin and complex conjugation symmetries).

It is convenient at this point to introduce notation to avoid confusion; from now on, we indicate within square brackets the particular set of alternative densities used in the TPSS correlation evaluation. Thus, DFT $[\varrho]$ refers to the deformed $\varrho_{\alpha}(r)$ and $\varrho_{\beta}(r)$ densities of PHF, DFT[ $\left.\rho\right]$ corresponds with $\rho_{>}$and $\rho_{<}$, and DFT $[u]$ uses $u_{A}$ and $u_{B}$. We remind the reader that DFT $[\rho]$ and DFT $[u]$ are constructed using the density matrix of the projected state.

\section{Computational details}

Our PHF+DFT scheme using alternative densities was implemented in the GAUSSIAN suite of programs. ${ }^{38} \mathrm{Un}$ less otherwise noted, all calculations in this work are carried out with the cc-pVTZ basis set. ${ }^{39}$ Unrestricted HartreeFock wavefunctions were used to generate initial guesses for the SUHF and KSUHF calculations. Frozen-core unrestricted coupled cluster singles and doubles with perturbative triplets, $\mathrm{UCCSD}(\mathrm{T}),{ }^{40}$ served as comparison reference in some examples. For KSUHF initial guesses, a complex phase factor was also applied to some elements of the HOMO orbital in order to break complex conjugation symmetry. Convergence extrapolation techniques ${ }^{41,42}$ (DIIS) were also applied when feasible. The Nelder-Mead algorithm ${ }^{43}$ was utilized for the optimization of the TPSS parameters.

\section{RESULTS AND DISCUSSION}

\section{A. Molecular dissociations}

Molecular dissociations make a good test for PHF+DFT as a quantitatively accurate description of this phenomenon demands the inclusion of both static and dynamic correlations. In this case, the overall best results are obtained by the least computationally demanding and simplest of the explored

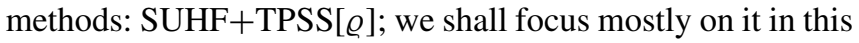
section.

Figure 2(a) shows excellent agreement between the dissociation curves of $\mathrm{H}_{2}$ computed from full-CI and SUHF+TPSS $[\varrho]$ with the optimized parameters $d=31.68$ and $C(0,0)=-0.22$. This figure also shows the UHF, SUHF, and UHF+TPSS curves for comparison. Note that addition of TPSS correlation yields a curve that tends to be parallel to the parent method, leading to a more accurate curve shape in SUHF+TPSS than in UHF+TPSS. The correlation energy $E_{c}^{\mathrm{TPSS}}\left[\varrho_{\alpha}, \varrho_{\beta}\right]$ in $\mathrm{H}_{2}$ tends to zero as the bond length increases, reflecting that the SUHF $\varrho_{\alpha}$ and $\varrho_{\beta}$ densities are compatible with TPSS and that this functional is free of selfinteraction error. We note that a curve nearly overlapping SUHF+TPSS $[\varrho]$ can also be obtained using SUHF+TPSS $[\rho]$ with $d=8.91$ and $C(0,0)=-0.79$. In contrast, it was not possible to achieve such a good agreement with FCI through all the dissociation region with SUHF+TPSS $[u]$ because $E_{c}^{\mathrm{TPSS}}\left[u_{A}, u_{B}\right]$ approaches zero more slowly than $E_{c}^{\mathrm{TPSS}}\left[\varrho_{\alpha}, \varrho_{\beta}\right]$ and $E_{c}^{\mathrm{TPSS}}\left[\rho_{>}, \varrho_{<}\right]$.

For larger systems, it is not viable to use full-CI to assess the accuracy of our method. However, UCCSD(T) has 

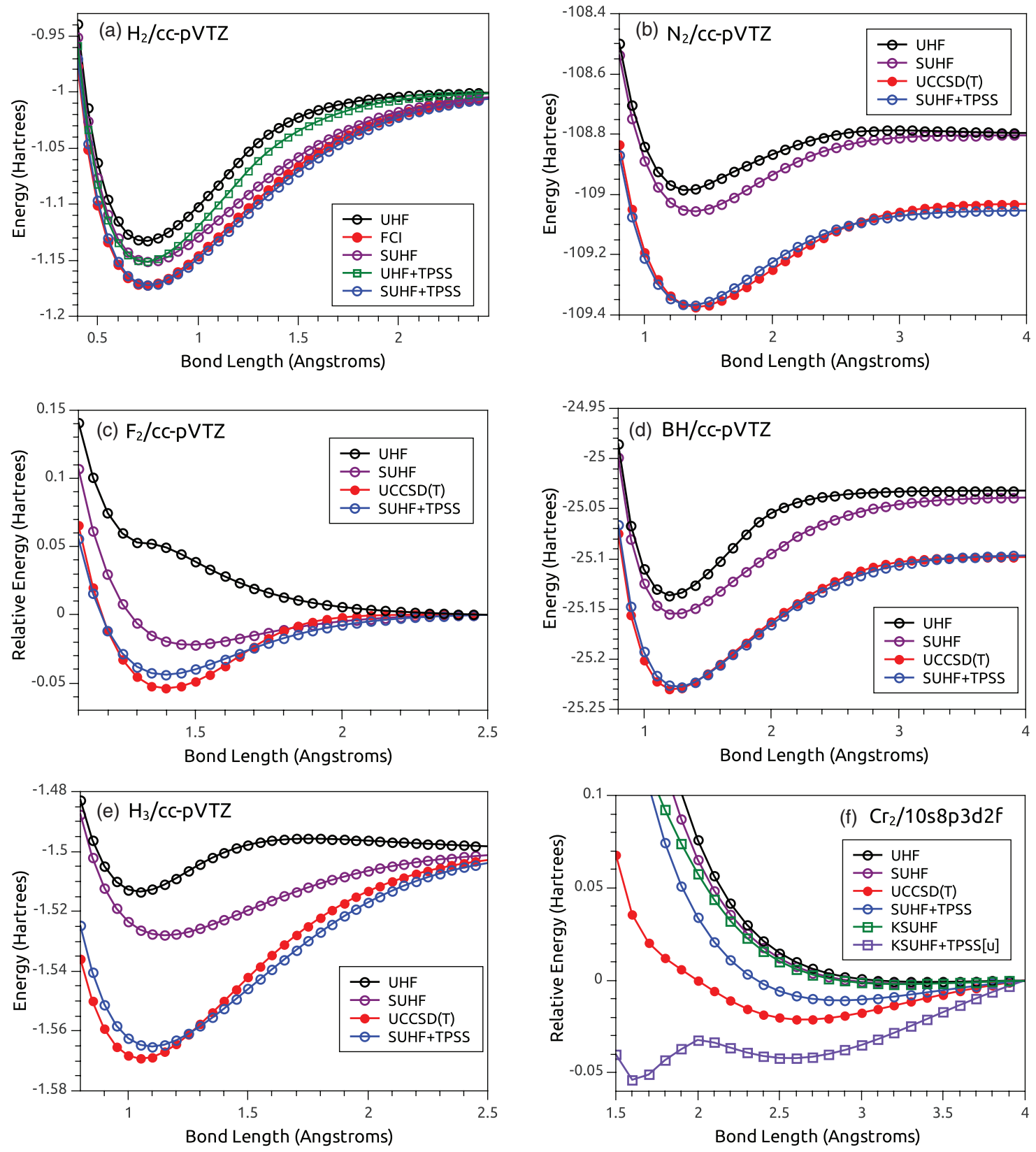

FIG. 2. Dissociation curves comparing results from SUHF+TPSS[@] with standard methods. All SUHF+TPSS calculations use $d=31.68$ and $C(0,0)$ $=-0.22$ while KSUHF+TPSS $[u]$ uses $d=1.21$ and $C(0,0)=12.28$.

been shown to provide potential energy curves very close to those of full-CI in some small molecules ${ }^{44}$ and so we use it as reference here. Using the same $d=31.68$ and $C(0,0)$ $=-0.22$ parameters, SUHF+TPSS $[\varrho]$ yields results very similar to those obtained from UCCSD(T) for the dissociations of $\mathrm{N}_{2}, \mathrm{~F}_{2}, \mathrm{BH}$, and triangular $\mathrm{H}_{3}$ (Figures 2(b)-2(e)). All these plots show total energies except for the $F_{2}$ dissociation curve (Figure 2(c)) because the amount of dynamic correlation is so large here that UHF and SUHF are on a different scale as compared to UCCSD(T) and SUHF+TPSS. These figures also demonstrate that SUHF can miss a large amount of dynamic correlation even for simple systems; however, SUHF energies can be greatly improved by addition of TPSS correlation with virtually no increase in computational cost.
The results are not quite as encouraging for the notoriously difficult case of the chromium dimer. Figure 2(f) shows the potential energy curve for $\mathrm{Cr}_{2}$ given by different methods using the $10 s 8 p 3 d 2 f$ basis of Scuseria and Schaefer. ${ }^{45} \mathrm{In} \mathrm{Cr}_{2}$, a mixture of $4 s-4 s$ and $3 d-3 d$ interactions with antiferromagnetic coupling results in a very short bond length but weak binding energy of $1.679 \AA$ and $34 \mathrm{kcal} / \mathrm{mol}$ ( 0.054 hartrees), respectively. ${ }^{46} \mathrm{UHF}$ and SUHF predict only a very weak binding in a region around 2.6-3.0 $\AA$, while SUHF+TPSS is nearly parallel to SUHF. The binding in this region is due to the $4 s-4 s$ interactions; the bonding at shorter distances due to $3 d-3 d$ interactions is completely absent in these dissociation curves. Nevertheless, the sophisticated, $\mathcal{O}\left(M^{7}\right), \operatorname{UCCSD}(\mathrm{T})$ method also fails to correctly describe the bonding in $\mathrm{Cr}_{2}$ (although better results have been reported using closed-shell 
$\operatorname{CCSD}(\mathrm{T})$ based on a sextuply bonded Slater determinant ${ }^{47}$ ). The chromium dimer is a notoriously difficult dissociation where both static and dynamic correlations have large contributions, thus it is not terribly surprising that the simple combinations proposed in this work are unsuccessful; it is estimated that traditional CI methods would require more than ten billion configurations to achieve high accuracy in $\mathrm{Cr}_{2}{ }^{48}$

In an attempt to fix this deficiency of SUHF+TPSS, we have also experimented with different alternative densities and the TPSS parameters. However, we were unable to find a combination of SUHF alternative densities and TPSS parameters that improved the description of the $\mathrm{Cr}_{2}$ dissociation. In fact, SUHF+TPSS $[\varrho]$, SUHF+TPSS $[\rho]$, and SUHF+TPSS $[u]$ all give very similar results and variation of $d$ and $C(0,0)$ always result in curves that are almost parallel to SUHF; variation of $d$ and $C(0,0)$ results mostly in a rescaling of $E_{c}^{\mathrm{TPSS}}$. It is possible, however, to obtain the qualitatively correct dissociation curve by incorporating complex conjugation symmetry breaking in the mixture. The KSUHF+TPSS $[u]$ curve shown in Figure 2(f) uses parameters optimized to fit the experimental dissociation energy of 0.054 hartrees $(d=1.21$ and $C(0,0)=12.28)$. Here, alternative densities $u_{A}$ and $u_{B}$ are used because TPSS does not work well with the deformed KSUHF densities, yielding a non-zero $E_{c}$ for one-electron densities. Apparently, breaking and restoring complex conjugation in addition to $\hat{S}^{2}$ symmetry, which essentially adds a $2 \times 2$ non-orthogonal $\mathrm{CI}$ to the wavefunction, allows KSUHF to capture correlations from a different spin reconfiguration, one with orbitals that are necessary for describing the dynamic correlations responsible for the $3 d-3 d$ bonding in the chromium dimer.

Unfortunately, KSUHF+TPSS is found to be inadequate for general use (at least in its current form) as it usually leads to serious overcorrelation problems. Clearly, what is needed in $\mathrm{Cr}_{2}$ (i.e., the inclusion of complex conjugation and a drastic change in the TPSS parameters) becomes double counting in non-metallic bonds. Modifications to $C(0,0)$ and $d$ do not fix the issue as using parameters optimized for a certain system can even lead to unphysical results (e.g., positive $E_{c}^{\text {TPSS }}$ ) in another system. Nonetheless, it may be possible to improve the general applicability of KSUHF+TPSS through a scheme that properly addresses the problem of double counting correlations.

\section{B. Singlet-triplet splittings}

Singlet-triplet splittings (the energy difference between the lowest singlet and triplet states) are particularly challenging to predict accurately because of their high sensitivity to both static and dynamic correlation effects. Table I compares the singlet-triplet splittings predicted by different approximations with experimental data for a set of particularly challenging benchmarks ${ }^{49-52}$ including small diatomics, ${ }^{53}$ methylene, ${ }^{54}$ trimethylenemethane, ${ }^{55}$ and benzyne isomers. ${ }^{56}$ Here, UHF+TPSS data are also given for comparison purposes; while it improves upon UHF, SUHF+TPSS techniques roughly halve the mean average error (MAE) of UHF+TPSS. The improvement given by SUHF+TPSS methods over SUHF is more moderate but significant; in particular, SUHF+TPSS predicts the correct sign for the splitting in each case, whereas SUHF fails for $m$-benzyne. Clearly, inclusion of dynamic correlation is important to describe this benzyne isomer, an observation in agreement with previous studies of spin splittings using unrestricted mean-field methods. ${ }^{49}$ The SUHF+TPSS singlet-triplet gaps are also more accurate than those reported for spin unrestricted calculations using state of the art DFT functionals. ${ }^{52}$ Nonetheless, the negative mean errors (MEs) indicate that SUHF+DFT tends to overcorrelate the singlet states, a problem that also seems to occur in other MR+DFT approaches. ${ }^{16}$

Although variation of the TPSS parameters has important effects on the total energies in SUHF+TPSS, the change in relative energies seems to be much less significant. Typically, use of the parameters $C(0,0)=-0.22$ and $d=31.68$ yields a correlation energy that is about half of the correlation energy using the default values of $C(0,0)=0.53$ and $d=2.8$. Nevertheless, the MAE in Table I for SUHF+TPSS[Q] using these different parametrizations are very close to each other. In fact, SUHF+TPSS[Q] with the default parameters gave the lowest MAE suggesting that one may use these parameters if interested only in relative energies. This is similar to what

TABLE I. Singlet-triplet splittings (in kcal/mol) for different approximations using the cc-pVTZ basis. The energies are given by $E_{S}-E_{T}$, ME is the mean error (theory - experiment), MAE the mean absolute error, and TMM denotes trimethylenemethane. The experimental data were taken from Refs. 53-56.

\begin{tabular}{|c|c|c|c|c|c|c|c|c|}
\hline Molecule & UHF & $\mathrm{UHF}+\mathrm{TPSS}[\varrho]^{\mathrm{a}}$ & SUHF & SUHF+TPSS $[\varrho]^{\mathrm{b}}$ & $\mathrm{SUHF}+\mathrm{TPSS}[\varrho]^{\mathrm{a}}$ & $\mathrm{SUHF}+\mathrm{TPSS}[u]^{\mathrm{a}}$ & $\mathrm{SUHF}+\mathrm{TPSS}[\rho]^{\mathrm{a}}$ & Expt. \\
\hline $\mathrm{NH}$ & 19.4 & 16.7 & 33.6 & 42.1 & 31.9 & 33.7 & 33.7 & 39.0 \\
\hline $\mathrm{OH}^{+}$ & 25.9 & 22.8 & 45.8 & 43.1 & 43.4 & 45.7 & 45.7 & 50.6 \\
\hline $\mathrm{O}_{2}$ & 15.8 & 15.6 & 20.6 & 22.9 & 21.7 & 22.3 & 22.4 & 22.6 \\
\hline $\mathrm{NF}$ & 19.7 & 16.7 & 32.3 & 35.5 & 32.6 & 31.7 & 31.8 & 34.3 \\
\hline $\mathrm{CH}_{2}$ & 16.9 & 13.1 & 15.6 & 17.9 & 12.8 & 10.0 & 9.8 & 9.4 \\
\hline TMM & 23.7 & 23.7 & 19.1 & 20.8 & 22.4 & 20.6 & 20.6 & 17.7 \\
\hline$o$-Benzyne & -15.8 & -20.6 & -51.4 & -53.3 & -51.6 & -62.5 & -62.9 & -38.0 \\
\hline$m$-Benzyne & 28.8 & 0.5 & 2.2 & -15.8 & -16.7 & -19.4 & -18.2 & -20.6 \\
\hline$p$-Benzyne & -10.1 & -6.2 & -28.2 & -25.0 & -21.8 & -28.8 & -31.5 & -3.5 \\
\hline ME & 1.3 & -3.24 & -2.43 & -2.59 & -4.08 & -6.47 & -6.68 & \\
\hline MAE & 17.5 & 13.96 & 9.19 & 7.26 & 6.75 & 7.51 & 7.94 & \\
\hline
\end{tabular}

${ }^{\mathrm{a}} C(0,0)=0.53$ and $d=2.8$.

${ }^{\mathrm{b}} C(0,0)=-0.22$ and $d=31.68$. 
occurs in DFT, where the total energies tend to be well below the variational limit, but the relative energies are very reasonable.

Another observation that can be drawn from Table I is that SUHF+TPSS $[\rho]$ and SUHF+TPSS $[u]$ yield very similar results. In the cases where the splittings are nearly identical, the occupation numbers of $\gamma\left(r, r^{\prime}\right)$ were such that $\rho_{<}$and $\rho_{>}$ were similar to $u_{A}$ and $u_{B}$. Also, the MAE does not change drastically when using the deformed $\varrho_{\alpha}$ and $\varrho_{\beta}$ densities. All this suggests that PHF+DFT is relatively insensitive to the type of alternative densities used. The densities $\varrho_{\alpha}$ and $\varrho_{\beta}$ may then be preferred since they do not require the computation of $\gamma\left(r, r^{\prime}\right)$ or any kind of transformations. However, if one uses a PHF method which breaks any kind of symmetry other than $\hat{S}^{2}$ symmetry, use of $\varrho_{\alpha}$ and $\varrho_{\beta}$ may introduce selfinteraction error, and in that case, $\rho_{<}$and $\rho_{>}$or $u_{A}$ and $u_{B}$ are likely to be a better choice.

\section{CONCLUDING REMARKS}

We have presented a simple method combining PHF and DFT correlation via alternative densities. The method has low mean-field computational cost and can handle several systems in which both dynamic and static correlations are present. Overall, SUHF+TPSS[@] gives the best results and seems to be more generally applicable (although it is not able to properly describe the most challenging case of the chromium dimer). Because of the large variety of plausible PHF+DFT combinations, there are still many alternatives to be explored, opening possibilities to improve upon the results reported here. We believe that PHF+DFT should be viewed as a low cost alternative within the multireference DFT family and therefore a promising candidate for treating large systems in which both types of correlation are important.

\section{ACKNOWLEDGMENTS}

This work was supported by the National Science Foundation CHE-1110884 and the Welch Foundation (C0036). A.J.G. acknowledges helpful discussions with Takashi Tsuchimochi.

${ }^{1}$ C. A. Jiménez-Hoyos, T. M. Henderson, T. Tsuchimochi, and G. E. Scuseria, J. Chem. Phys. 136, 164109 (2012).

${ }^{2}$ D. L. Strout and G. E. Scuseria, J. Chem. Phys. 102, 8448 (1995).

${ }^{3}$ K. Burke, J. Chem. Phys. 136, 150901 (2012) and references therein.

${ }^{4}$ G. C. Lie and E. Clementi, J. Chem. Phys. 60, 1275 (1974).

${ }^{5}$ G. C. Lie and E. Clementi, J. Chem. Phys. 60, 1288 (1974).

${ }^{6}$ R. Colle and O. Salvetti, Theor. Chim. Acta 37, 329 (1975).

${ }^{7}$ R. Colle and O. Salvetti, Theor. Chim. Acta 53, 55 (1979)

${ }^{8}$ F. Moscardó and E. San-Fabián, Phys. Rev. A 44, 1549 (1991).

${ }^{9}$ E. Kraka, Chem. Phys. 161, 149 (1992).

${ }^{10}$ T. Leininger, H. Stoll, H. Werner, and A. Savin, Chem. Phys. Lett. 275, 151 (1997).
${ }^{11}$ B. Miehlich, H. Stoll, and A. Savin, Mol. Phys. 91, 527 (1997).

${ }^{12}$ J. Gräfenstein and D. Cremer, Chem. Phys. Lett. 316, 569 (2000).

${ }^{13}$ R. Takeda, S. Yamanaka, and K. Yamaguchi, Chem. Phys. Lett. 366, 321 (2002).

${ }^{14}$ J. Gräfenstein and D. Cremer, Mol. Phys. 103, 279 (2005).

${ }^{15}$ R. Pollet, A. Savin, T. Leininger, and H. Stoll, J. Chem. Phys. 116, 1250 (2002).

${ }^{16}$ A. J. Pérez-Jiménez and J. M. Pérez-Jordá, Phys. Rev. A 75, 012503 (2007).

${ }^{17}$ T. Tsuchimochi and G. E. Scuseria, J. Chem. Phys. 131, 121102 (2009).

${ }^{18}$ T. Tsuchimochi, G. E. Scuseria, and A. Savin, J. Chem. Phys. 132, 024111 (2010).

${ }^{19}$ J. P. Perdew, A. Savin, and K. Burke, Phys. Rev. A 51, 4531 (1995).

${ }^{20}$ W. Kohn and L. J. Sham, Phys. Rev. 140, A1133 (1965).

${ }^{21}$ P. Hohenberg and W. Kohn, Phys. Rev. 136, B864 (1964).

${ }^{22} \mathrm{~F}$. Jensen, Introduction to Computational Chemistry, 2nd ed. (Wiley, England, 2007) p. 246

${ }^{23}$ T. Ziegler and A. Rauk, Theor. Chim. Acta 43, 261 (1977).

${ }^{24}$ K. Yamaguchi and T. Fueno, Chem. Phys. 19, 35 (1977).

${ }^{25}$ A. D. Becke, A. Savin, and H. Stoll, Theor. Chim. Acta 91, 147 (1995).

${ }^{26}$ G. E. Scuseria, C. A. Jiménez-Hoyos, T. M. Henderson, K. Samanta, and J. K. Ellis, J. Chem. Phys. 135, 124108 (2011).

${ }^{27}$ A. J. Pérez-Jiménez and J. M. Pérez-Jordá, J. Chem. Phys. 120, 18 (2004).

${ }^{28}$ K. Takatsuka, T. Fueno, and K. Yamaguchi, Theor. Chim. Acta 48, 175 (1978).

${ }^{29}$ V. N. Staroverov and E. R. Davidson, Chem. Phys. Lett. 340, 142 (2001).

${ }^{30}$ M. Head-Gordon, Chem. Phys. Lett. 372, 508 (2003).

${ }^{31}$ A. Zunger and J. P. Perdew, Phys. Rev. B 23, 5048 (1981).

${ }^{32}$ O. A. Vydrov, Ph.D. dissertation, Rice University, 2007.

${ }^{33}$ O. A. Vydrov and G. E. Scuseria, J. Chem. Phys. 121, 8187 (2004).

${ }^{34}$ O. A. Vydrov, G. E. Scuseria, J. P. Perdew, A. Ruzsinszky, and G. I. Csonka, J. Chem. Phys. 124, 094108 (2006).

${ }^{35}$ J. P. Perdew, K. Burke, and Y. Wang, Phys. Rev. B 54, 16533 (1996).

${ }^{36}$ J. P. Perdew, K. Burke, and M. Ernzerhof, Phys. Rev. Lett. 77, 3865 (1996); 78, 1396(E) (1997).

${ }^{37}$ J. Tao, J. P. Perdew, V. N. Staroverov, and G. E. Scuseria, Phys. Rev. Lett. 91, 146401 (2003)

${ }^{38}$ M. J. Frisch, G. W. Trucks, H. B. Schlegel et al., GAUSSIAN 09, Development Version, Revision H.1, Gaussian Inc., Wallingford, CT, 2009.

${ }^{39}$ T. H. Dunning, J. Chem. Phys. 90, 1007 (1989).

${ }^{40}$ J. A. Pople, M. Head-Gordon, and K. Raghavachari, J. Chem. Phys. 87, 5968 (1987).

${ }^{41}$ P. Pulay, Chem. Phys. Lett. 73, 393 (1980).

${ }^{42}$ G. E. Scuseria, T. J. Lee, and H. F. Schaefer, Chem. Phys. Lett. 130, 236 (1986).

${ }^{43}$ J. A. Nelder and R. Mead, Comput. J. 7, 308 (1965).

${ }^{44}$ A. Dutta and C. D. Sherrill, J. Chem. Phys. 118, 1610 (2003).

${ }^{45}$ G. E. Scuseria and H. F. Schaefer, Chem. Phys. Lett. 174, 501 (1990).

${ }^{46}$ E. J. Thomas III, J. S. Murray, C. J. O'Connor, and P. Politzer, THEOCHEM 487, 177 (1999).

${ }^{47}$ G. E. Scuseria, J. Chem. Phys. 94, 442 (1991)

${ }^{48}$ S. R. Langhoff and C. W. Bauschlicher, Ann. Rev. Phys. Chem. 39, 181 (1988).

${ }^{49}$ T. Tsuchimochi and G. E. Scuseria, J. Chem. Phys. 134, 064101 (2011).

${ }^{50}$ L. V. Slipchenko and A. I. Krylov, J. Chem. Phys. 117, 4694 (2002).

${ }^{51}$ T. Saito, S. Nishihara, S. Yamanaka, Y. Kitagawa, T. Kawakami, S. Yamada, H. Isobe, M. Okumura, and K. Yamaguchi, Theor. Chem. Acc. 130, 739 (2011).

${ }^{52}$ D. H. Ess and T. C. Cook, J. Phys. Chem. A 116, 4922 (2012).

${ }^{53}$ K. P. Huber and G. Herzberg, Constants of Diatomic Molecules (Van Nostrand Reinhold, New York, 1979).

${ }^{54}$ P. Jensen and P. R. Bunker, J. Chem. Phys. 89, 1327 (1988).

${ }^{55}$ P. G. Wenthold, J. Hu, R. R. Squires, and W. C. Lineberger, J. Am. Chem. Soc. 118, 475 (1996).

${ }^{56}$ P. G. Wenthold, R. R. Squires, and W. C. Lineberger, J. Am. Chem. Soc. 120, 5279 (1998). 